\title{
Determinants of variations in breast milk protective factor concentrations of rural Gambian mothers
}

\author{
A PRENTICE, A M PRENTICE, T J COLE, AND R G WHITEHEAD \\ Medical Research Council, Dunn Nutrition Unit, Cambridge, and Keneba, The Gambia
}

SUMMARY The concentrations of 7 immunoproteins (IgA, IgG, IgM, the complement components $\mathrm{C} 3$ and C4, lactoferrin, and lysozyme) in the breast milk of 152 rural west African women were measured as part of a semilongitudinal study to assess their importance in infant health. Each mother maintained a characteristic level of production of immunoproteins relative to other mothers, and the concentration of each immunoprotein within each woman was correlated positively with the others. Parity was the major determinant of ranking and mothers of parity 1 and 2 produced the highest concentrations of immunoproteins. Except for lysozyme, infants' intake of these protective factors decreased in early lactation, but infants aged 1-2 years still received substantial amounts. The daily intakes by Gambian infants were similar to or higher than those of infants in Cambridge, United Kingdom. A marked seasonal increase in infant morbidity was not accompanied by an increase in the concentrations of protective factors in mothers' milk.

Although our knowledge of the antimicrobial capacity of human milk advances rapidly, we know little about the practical contribution to the health of the breast fed infant of the protective factors in milk. ${ }^{1}$ This is of particular relevance in the developing world where breast feeding is sustained for long periods and where infants usually grow up in a heavily contaminated environment. We undertook a semilongitudinal study of the concentrations of 7 major immunoproteins in the milk from rural Gambian women. Several determinants of the concentrations of these protective factors (PF) were identified, and the size and consistency of the variations suggested that different health patterns among infants may be partly ascribed to differences in their PF intakes.

\section{Methods}

Subjects. All lactating women of more than 14 days postpartum in the neighbouring villages of Keneba and Manduar in The Gambia took part in this study. In this community women breast feed for at least 18 months and data have been obtained up to 26 months' lactation. Milk samples were collected at 3 different times of the year, chosen to represent periods of varying nutritional and infective stress on the mother and her infant. These sampling periods were:

April-May Dry season, low prevalence of infections, good maternal food intake, light $/ \mathrm{mod}$ erate farm work.
June-July Beginning of the rainy season, increasing prevalence of infections, declining food stocks, very heavy farm work.

August-September Middle/end of the rainy season, high prevalence of infections in both mothers and infants, poor food stocks, moderate/ heavy farm work.

Ninety of the 152 mothers in the study were receiving an energy rich dietary supplement described elsewhere. ${ }^{2}$ Twelve hour breast milk intakes of 95 infants were also measured up to 18 months of age by test weighing on 2 occasions in every sampling period. ${ }^{3}$ Full obstetric histories of each mother were established. For comparison similar measurements were carried out on the milks of 10 women in Cambridge. The study was approved by the ethical committees of the Dunn Nutrition Unit, Cambridge and of the Medical Research Council Laboratories, The Gambia.

Sample collection and analysis. Milk samples $(0.5 \mathrm{ml})$ collected from each breast by manual expression between 8 am and 10 am were stored at $-20^{\circ} \mathrm{C}$. The milks were analysed for IgA, IgG, IgM, lactoferrin, lysozyme, and 2 of the complement components, $\mathrm{C} 3$ and $\mathrm{C} 4$, by single radial immunodiffusion. ${ }^{4}$ Standardisation was achieved using control human sera (Hyland, Behringwerke), human lactoferrin (Calbiochem-Behring), and human lysozyme (courtesy of Professor E F Osserman). Milk IgA values have been expressed in arbitrary 
units as secretory and serum IgA are not antigenically equivalent.

Statistical analysis. Before statistical analysis the PF concentrations were logarithmically transformed and the values for each breast averaged. Comparison of data from more than one sampling period was made using the method of Tanner for mixed longitudinal series. ${ }^{5}$ This allowed for more than 1 measurement per mother. Tests of significance were performed using step wise multiple regression analysis, one way analysis of variance, and Student's $t$ test, as appropriate.

\section{Results}

Variation with stage of lactation. All PF concentrations, with the exception of lysozyme, decreased during the first year of lactation. No further decrease occurred thereafter and with some PF, notably IgA, a rise in concentration was seen in the second year (Fig. 1 (a) and (c)). In contrast, the concentration of

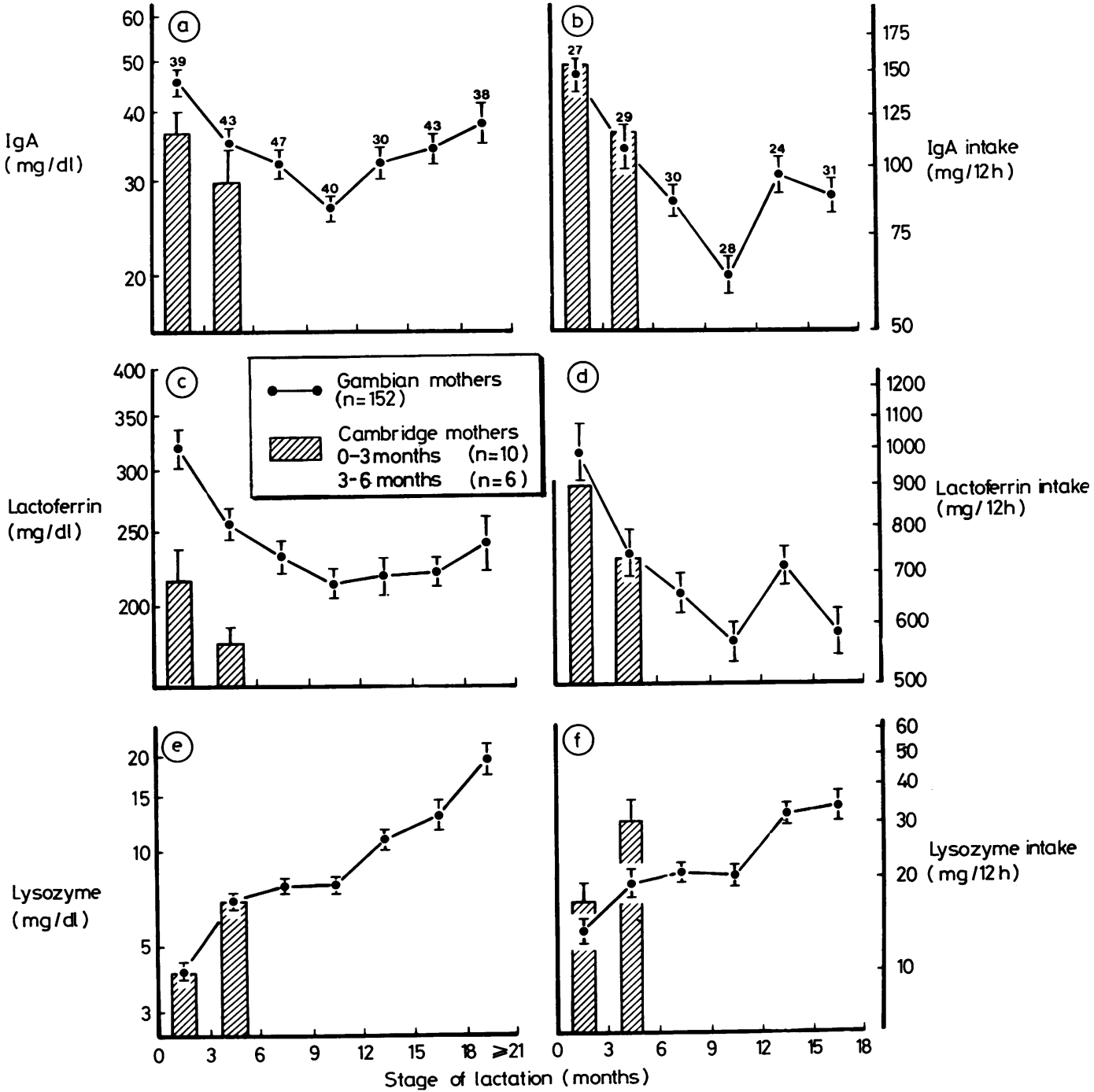

Fig. 1 Examples of the effect of the stage of lactation on milk protective factor concentrations and 12 hour intakes. Values represent $\log _{10}$ (geometric mean $\pm S E M$ ). Numbers of Gambian mothers at each stage of lactation are shown in the top portion of the diagram. 
lysozyme rose progressively throughout lactation and was, on average, 4.7 times higher in the latter part of the second year postpartum than in the first 3 months of lactation (Fig. 1(e)). Because of a concomitant decrease in breast milk volume after 2-3 months postpartum that is a feature of this community, ${ }^{3} 12$ hour intakes of PF, except for lysozyme, fell sharply during the first year. PF intakes were then maintained for up to 18 months lactation at least, suggesting that some degree of compensation for the declining breast milk volume occurred (Fig. 1 (b) and (d)). The 12 hour intake of lysozyme rose throughout lactation and the increase in concentration more than offset the decrease in breast milk volume (Fig. 1 (f)).

Seasonal variation. In the season of peak prevalence of diarrhoea and many other infectious diseases (August-September) ${ }^{6} 7$ no increase was observed in the concentrations of any PF. Indeed there were appreciable decreases in IgA, IgG, C4, and lysozyme concentrations between milk collected in JuneJuly and August-September (Fig. 2). It is possible, however, that the spectrum of specific immunoglobulins responded to new infections even though the total concentration decreased. As breast milk volumes of Gambian mothers also decrease during

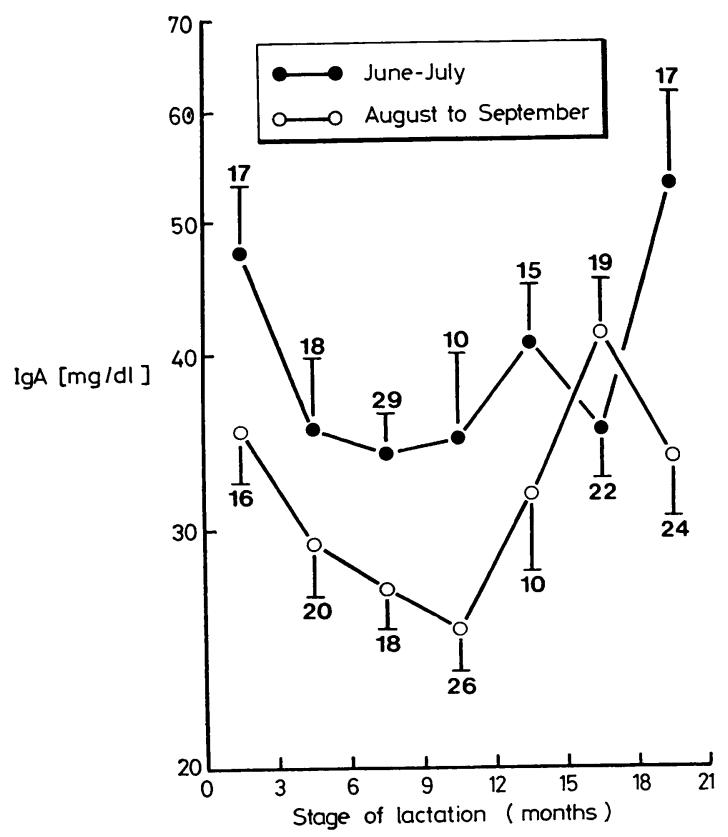

Fig. 2 Effect of season on milk IgA concentrations. Values represent $\log _{10}$ (geometric mean $\pm S E M$ ). the rainy season ${ }^{3}$ the 12 hour intakes of all PF were much lower at this time of year. There was, for example, an average decrease of $25 \%$ in 12 hour IgA intake at each stage of lactation between the beginning and the end of the rainy season.

Variation with plane of nutrition. A comparison between mothers who received dietary supplements and those who did not showed no important differences in the concentrations of any PF at any time, despite the very substantial difference in food intake (mean maternal energy intakes of 2300 $\mathrm{kcal} /$ day $(9.6 \mathrm{MJ} /$ day) and 1650 to $1200 \mathrm{kcal} /$ day (6.9 to $5.0 \mathrm{MJ} /$ day) respectively $\left.{ }^{2}\right)$. The normal diet in this community is not, however, substantially protein deficient and it is possible that PF values may be influenced by severe maternal protein deficiency.

Comparison with Cambridge milks. The 12 hour intakes of IgA and lactoferrin were identical in Cambridge and The Gambia (Fig. 1 (b) and (d)), since the slightly lower volumes of breast milk produced by the Gambian mothers were offset by higher concentrations of these PF in their milk (Fig. 1 (a) and (c)). Milk lysozyme concentrations were similar in the 2 communities leading to slightly raised 12 hour lysozyme intakes in Cambridge (Fig. 1 (e) and (f)).

In contrast, both the concentrations and 12 hour intakes of the other 4 PF (IgG, IgM, C3, and C4) were higher in The Gambia compared with Cambridge (not illustrated). Since these factors may well be derived directly from the bloodstream, this difference between the 2 communities is probably a consequence of the well documented raised concentrations of immunoproteins in the plasma of west Africans compared with Caucasians. ${ }^{89}$

Consistency of PF concentrations within individuals. One way analysis of variance on data, standardised for season and stage of lactation, over the 3 sampling periods $(\mathrm{P}<0.001$ for each $\mathrm{PF})$ showed that individual women exhibited a characteristic level of production of each PF. Moreover, a similar analysis of these corrected values showed that there was a strong positive interrelation between all the $\mathrm{PF}$ concentrations in the milk of each subject $(F=6.84$ on 912 and $151 \mathrm{df}, \mathrm{P}<0.001)$. Thus an individual who had milk that was comparatively rich in one PF usually had raised values of all other PF relative to other mothers in the community, and this differential was maintained throughout the 3 sampling periods.

Effect of parity. Parity of the mother was the major determinant of her characteristic level of PF production. Relative total PF values, derived by taking the 


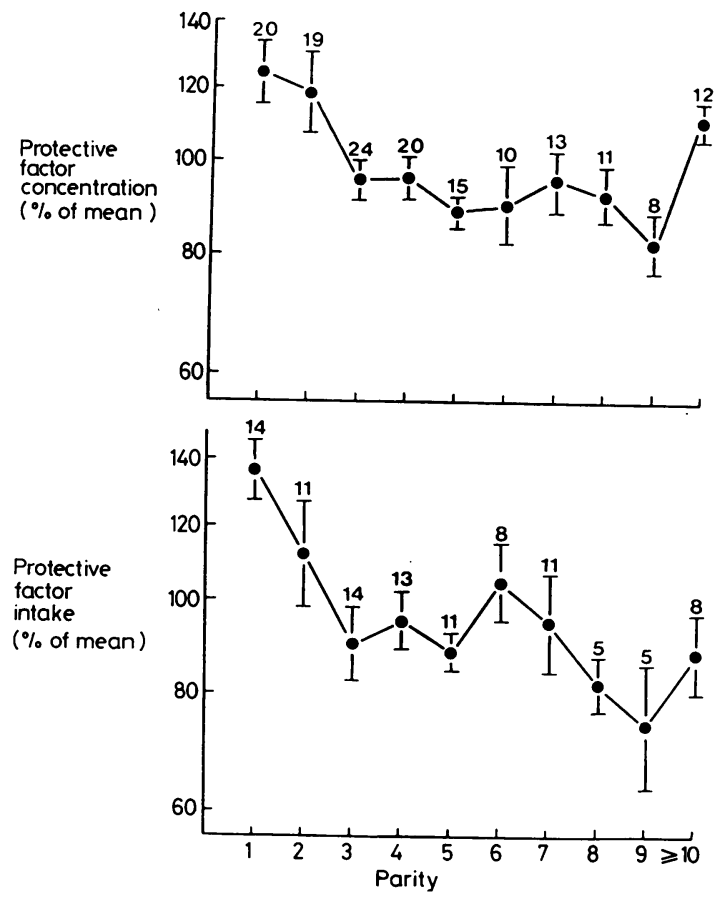

Fig. 3 Effect of parity on protective factor $(P F)$ concentrations and 12 hour intakes. Values represent $\log _{10}$ (geometric mean $\pm S E M$ ) after standardisation for season and stage of lactation and using a combined measurement of all PF for each individual.

mean of all corrected PF concentrations for each woman, were significantly higher in mothers of parity 1 and 2 compared with those of parity 3 to 9 $(\mathrm{P}<0.001)$ and there was a second rise at and beyond parity $10(P<0.01)$ (Fig. 3). Similar patterns were obtained for each PF analysed separately. The 12 hour breast milk volumes from the subset of mothers for whom volumes were available were unaffected by parity once season and stage of lactation were taken into account except for a significant drop at parities $\geqslant 9(\mathrm{P}<0.02)$. Consequently, the relative 12 hour output of PF was significantly higher for mothers of parity 1 and 2 than for higher parities $(P<0.001)$ but there was no rise in the PF concentrations in milks of mothers of very high parity (Fig. 3). It can be estimated that, on average, a first born child will receive $40 \%$ more of all the PF throughout lactation compared with a child born at the same time to a mother of parity $\geqslant 8$.

\section{Discussion}

Two findings of major importance have emerged from this study. The first is that substantial long term differences exist between the PF outputs of different women. The major determinant of a woman's immunosecretory ability is parity. When discussing the merits of breast feeding we should be aware that the infants of young mothers of low parity are likely to have a considerable advantage over those of multiparous women, who constitute the major portion of the child bearing population in developing countries.

The second important finding is that the intake of PF by all the infants decreased as the prevalence of infectious diseases, especially diarrhoea, increased in the rainy season. For some PF this reduction was caused by the decrease in milk volume at this time of year, but for IgA, IgG, C4, and lysozyme there were also substantial decreases in the milk concentration. An apparent failure to maintain protection at this most critical time of year may be a reflection of the extreme stresses to which Gambian women are subjected. It appears, however, that the reduction in maternal food intake that occurs in the rainy season was not responsible for the decreased PF outputs since similar changes were observed in the diet supplemented and unsupplemented groups. This apparent insensitivity of PF values to the plane of nutrition is further emphasised by the fact that at no time were the PF intakes of Gambian infants appreciably lower than those in Cambridge. Indeed the intakes of the passively transferred serum proteins were higher in the Gambian infants.

The decrease in concentration of most of these PF in mature milk during the first months of lactation has been well documented. ${ }^{10-13}$ This study shows however, that after the early decrease, PF values are well maintained until the end of lactation. Lysozyme differs from all the other immunoproteins in showing a progressive rise in concentration throughout lactation that more than compensates for the decreasing milk output. Such an increase has been noted in other communities that practice prolonged lactation,,$^{10} 13$ but no cause has yet been suggested. Gambian infants in the second year of life still receive considerable quantities of these immunoproteins, particularly of lysozyme, from their mother and these may continue to exert a protective action.

This study shcws that the concentrations of 7 major immunoproteins in mature milk are influenced by a number of factors other than the immediate environment. If it is assumed that these substances do have antimicrobial activities in vivo then it is clear that the size and consistency of the differences between individuals are such that certain children within a community will be less well protected against infections than others throughout infancy. We are currently testing this hypothesis in The 
Gambia by comparing longitudinally the morbidity patterns and PF intakes of individual infants.

We thank the field staff of the Dunn Nutrition Unit, Keneba, The Gambia, for assistance and Miss A A Paul for obtaining the milks and breast milk volumes of the Cambridge subjects.

\section{References}

1 Anonymous. The how of breast milk and infection. Lancet 1981 ; i: 1192-3.

2 Prentice AM, Whitehead RG, Roberts SB, et al. Dietary supplementation of Gambian nursing mothers and lactational performance. Lancet 1980; ii: 886-8.

3 Whitehead RG, Rowland MGM, HuttonM, Prentice AM, Muller EM, Paul AA. Factors influencing lactation performance in rural Gambian mothers. Lancet 1978; ii: 178-81.

4 Mancini G, Carbonara AO, Heremans JF. Immunochemical quantitation of antigens by single radial immunodiffusion. Immunochemistry 1965; 2: 235-54.

5 Tanner JM. Some notes on the reporting of growth data. Hum Biol 1951; 23: 93-159.

- McGregor IA, Rahman AK, Thomson AM, Billewicz WZ, Thompson B. The health of young children in a West African (Gambian) village. Trans $R$ Soc Trop Med Hyg 1970; 64: 48-77.

7 Rowland MGM, Barrell RAE. Ecological factors in gastroenterit1s. Proc Soc Study Hum Biol 1980; 7: 21-35.
${ }^{8}$ Logie DE, McGregor IA, Rowe DS, Billewicz WZ. Plasma immunoglobulin concentrations in mothers and newborn children with special reference to placental malaria. Bull WHO 1973; 49: 547-54.

9 Rowe DS, McGregor IA, Smith SJ, Hall P, Williams K. Plasma immunoglobulin concentrations in a West African (Gambian) community and in a group of healthy British adults. Clin Exp Immunol 1968; 3: 63-79.

10 Goldman AS, Garza C, Nichols BL, Goldblum RM. Immunologic factors in human milk during the first year of lactation. J Pediatr 1982; 100: 563-7.

11 Lönnerdal B, Forsum E, Gebre-Medhin M, Hambraeus L. Breast-milk composition in Ethiopian and Swedish mothers. II. Lactose, nitrogen, and protein contents. Am J Clin Nutr 1976; 29: 1134-41.

12 Peitersen B, Bohn L, Anderson H. Quantitative determination of immunoglobulins, lysozyme, and certain electrolytes in breast milk during the entire period of lactation, during a 24-hour period and in milk from the individual mammary gland. Acta Paediatr Scand 1975; 64: 709-17.

13 Reddy V, Bhaskaram C, Raghuramulu N, Jagadeesan V. Antimicrobial factors in human milk. Acta Paediatr Scand 1977; 66: 229-32.

Correspondence to Dr Ann Prentice, Dunn Nutrition Unit, Milton Road, Cambridge.

Received 30 November 1982. 